\title{
Improving the provision of driving advice on discharge after abdominal surgery
}

Imogen Buss, Laura Gould

North Bristol NHS Trust

\begin{abstract}
Ensuring the safety of patients is a vital duty of a doctor. It is their responsibility to advise patients about activity limitations on discharge from hospital. This study aims to assess the current provision of driving advice for patients after abdominal surgery and institute improvements to this provision of information in North Bristol NHS Trust.
\end{abstract}

A preliminary questionnaire ascertained current doctor's knowledge regarding limitations of driving postoperatively and whether information was communicated to patients. Baseline retrospective data were collected from electronic discharge summaries to determine documentation of advice provision. Educational interventions were introduced, followed by data collection after each intervention.

Initial questionnaires demonstrated poor knowledge amongst doctors and a lack of provision of driving advice postoperatively. After multiple educational interventions, the provision of driving advice on electronic discharge summaries increased from $0 \%(0)$ at baseline to $75 \%(9)$.

Initially, the provision of driving advice postoperatively was poorly documented for inpatients undergoing abdominal surgery; following multiple educational interventions, the provision of written advice improved. Future plans include the introduction of prewritten sentences onto the electronic discharge summaries to facilitate ease of information provision and a reaudit in 12 months.

\section{Problem}

Ensuring safety of patients is a vital responsibility of a doctor, both during their hospital stay and after discharge. It has been noticed that the provision of advice on discharge following surgery tends to vary in detail and documentation. We wanted to measure the provision of driving advice on discharge for patients following abdominal surgery. Provision of driving advice is vital to ensure the safety not only of the patients themselves, but also other road users.

The General Medical Council recommends in 'Good Medical Practice' that documents must be clear, accurate, and legible, and indicate that clinical records should include the information given to patients.[1] Evidently, if documentation is poor, it cannot be relied upon in the future if there were to be a legal dispute regarding clinical advice given.

In North Bristol NHS Trust, it was apparent from our clinical experience that patients were not receiving advice regarding driving on discharge from hospital, and the junior doctors were not aware of what information they should be providing.

\section{Background}

Ensuring a patient's safety is a vital responsibility of a doctor, and this does not solely refer to the time spent in hospital, but also for the recovery period after discharge.[1] Therefore we can assimilate that providing adequate driving advice to protect both patients and other members of the general public after discharge following surgery is a requirement of medical professionals. The DVLA current guidance is that drivers only need to inform the DVLA if a medical condition persists for longer than three months after surgery that would affect their ability to drive safely.[2] Despite it being the driver's responsibility to inform the DVLA, it is the doctor's responsibility to advise when it will be safe to return to driving after surgery.[2]

Current research investigating the provision of driving advice after caesarean section demonstrates that women are receiving conflicting advice and highlight a need for UK national guidelines for postoperative driving.[3,4] Similar issues seem to arise with advice regarding orthopaedic surgery,[5] although some research emphasises a need to individualise advice and highlight the importance of practising in a stationary car prior to driving.[6] Other evidence highlights the dramatic financial costs of providing inaccurate advice about returning to work after surgery.[7] However there is little literature investigating the provision of postoperative driving advice after abdominal surgery.

The current recommendations from the Royal College of Surgeons state that prior to resuming driving, one should be free from the sedative effects of analgesia, pain free, and able to perform an emergency stop.[8] However, regarding abdominal surgery, direct advice only exists for cholecystectomy (avoid for one week) and hernia repairs (at least one week).[8]

It is apparent that junior doctors are poorly informed about what driving advice to give and whose clinical responsibility it is to ensure 
BMJ Quality Improvement Reports

that this information is provided. Advice is often given verbally, and either not documented or poorly documented, contrary to recommendations.[9] It is well evidenced that patients do not retain all information provided to them whilst in hospital; providing written information in addition to verbal advice is paramount. An appropriate place to document such information would be on the discharge summary, in accordance with the GMC recommendations of clear and legible record keeping and documenting patient advice.[10] A previous Quality Improvement project in an acute stroke service demonstrated an improvement with their intervention from $3 \%$ to $79 \%$ of discharge summaries having driving advice documented.[11]

The primary aim of this quality improvement project was to analyse the current provision of driving advice on discharge following admission with abdominal surgery. The secondary aim was to encourage more thorough provision of driving advice on the electronic discharge summaries.

\section{Baseline measurement}

Two baseline measures were decided upon.

Firstly, an initial questionnaire was conducted [appendix 1] to ascertain the junior doctors' knowledge regarding the limitations on driving postoperatively and whether they were providing such information to patients routinely.

Ten responses were obtained. Eight did not provide advice on discharge; two provided verbal advice only, with only one documenting this. Nine respondents were not aware of any guidelines or recommendations; one was aware, but only for day surgery. Six respondents were aware of the conditions required before driving postoperatively such as being able to perform an emergency stop. All ten respondents did not know what to advise different patients depending on their operation, and all thought it would be useful to have prewritten advice box on electronic discharge summary for post operative driving advice.

Secondly, retrospective data were collected anonymously by recording all discharges from the three general surgical wards in Frenchay Hospital from 8am 22nd January to 8am on 29th January 2014. Discharges were noted from the discharge book and recorded on a spreadsheet [appendix 2].

The discharge summaries for these patients were then scrutinised. It was noted whether they underwent abdominal surgery during their admission and if so, whether driving advice was recorded on their discharge summary. The operation type, data and length of post operative stay was also recorded. Patients with prolonged post operative admissions ( $>35$ days), those under the legal age for driving and patients who were bed bound on discharge were excluded from the results.

Nineteen patients were discharged home after abdominal surgery during this time period, with none of them $(0 \%)$ having driving advice documented on the discharge summary.
See supplementary file: ds4755.docx - "APPENDICES 1+2"

\section{Design}

We decided that it was important to educate the clinical staff who complete the discharge summaries about the current guidelines and recommendations that they should be providing to patients on discharge from hospital.

Posters [appendix 3] were designed and printed regarding the provision of driving advice on discharge summaries. These were posted throughout the surgical department offices and ward as well as being emailed to all junior doctors on the surgical rotation.

Further interventions included emailing prewritten sentences providing driving advice [appendix 4] that could be copied into discharge summaries.

It was aimed to eventually instigate the provision of these prewritten sentences into the discharge summary boxes on the electronic discharge summaries. However, due to the move of two hospital sites into one new site during the data collection period, the Trust was unwilling to undertake new alterations at this busy time.

\section{Strategy}

PDSA Cycle 1. Posters [appendix 3] were designed with information regarding postoperative driving advice. They were designed to be eye catching and highlight the importance of providing information as well as what information to provide. They were displayed in the general surgical office, on the surgical wards and also emailed to the surgical junior doctors.

Following this a second data collection round was undertaken from 24th February-3rd March 2014, in the same manner as the baseline data collection.

PDSA Cycle 2. The second cycle occurred when the next rotation of junior doctors joined the general surgical department. Copies of the poster were placed in each doctors pigeon hole. In addition, an email explaining the junior doctors role in providing postoperative advice to patients was disseminated, which included specific driving advice paragraphs [appendix 4]. The third round of data collection was carried out between 21st-28th April 2014.

PDSA Cyle 3. Further education was provided to junior doctors both by email and face to face to reiterate the importance of advising postoperative patients about driving. The timing of this cycle coincided with the 'Advice to patients' section on the electronic discharge summaries becoming compulsory. Fourth round of data collection was from 23rd-30th June 2014.

See supplementary file: ds4754.docx - "APPENDICES 3+4"

\section{Post-measurement}

Baseline data demonstrated that there was a clear requirement for 


\section{BMJ Quality Improvement Reports}

an improvement in education among junior medical staff regarding the provision of driving advice for patients after abdominal surgery. $80 \%$ of respondents did not provide driving advice on discharge, with $90 \%$ not being aware of any guidelines or recommendations. Despite $60 \%$ knowing about the requirement of being able to perform an emergency stop prior to driving, $100 \%$ of respondents felt that the inclusion of pre-written driving advice on the discharge summaries would be a useful addition. Regarding the 19 patients undergoing abdominal surgery in the baseline data collection, $0 \%$ received driving advice on their discharge summaries.

Following the dissemination of posters highlighting the recommendations for the provision of driving advice via email and display around the general surgical offices (PDSA Cycle 1), the provision of driving advice on discharge summaries remained $0 \%$.

The second intervention consisted of presenting the driving recommendations at the surgical induction for the new cohort of junior doctors and emailing prewritten sentences that they could copy and paste into discharge summaries. This greatly improved the provision of driving advice on discharge summaries, from 0 to $36 \%$ (8 patients).

A final intervention included reiteration of the recommendations by email and face to face, coinciding with the "advice given to patients" section of the electronic discharge summary becoming compulsory. This further improved the provision of driving advice to $75 \%$ (9 patients). This is clearly a large improvement from $0 \%$ but further work is required to reach the target of $100 \%$.

See supplementary file: ds6105.docx - "Graphic Results QIP"

\section{Lessons and limitations}

The intervention used in this project focused on education of doctors and due to the rapid turnover of junior doctors on surgical rotations, it was difficult to implement an ongoing change. However, when the cohort changed and the intervention was widely discussed during their induction, we obtained much more complete documentation of driving advice. A more sustainable intervention would be the introduction of prewritten sentences on the electronic discharge summaries, as initially planned. It was not possible to introduce this intervention in the current time period due to the move of the hospitals from two sites to one during the year.

During the data collection, it was noted that some of the patients being discharged from hospital following abdominal surgery would not drive anyway, for example young patients who have not yet passed their test and those who no longer drive. Therefore it would be impossible to obtain $100 \%$ of patients receiving driving advice on discharge, as this is not relevant to all patients. A suggested solution would be to document in the discharge summary if they do not drive, thereby no advice regarding driving was provided.

Limitations from this project include the small numbers involved at each stage of data collection make it difficult to draw reliable conclusions from the results, however there is evidently an improvement in the provision of driving advice throughout the process.

The introduction of the mandatory 'Advice given to patients' box on the electronic discharge letter is likely to have prompted doctors to record driving advice. This implies that if the option for prewritten driving advice was available on a drop down menu it would be widely used.

\section{Conclusion}

There is evidently a lack of education among junior doctors regarding the current guidelines and recommendations for the provision of driving advice on discharge for surgical patients. Our educational interventions have helped to improve the provision and documentation of such advice. Ongoing education of each cohort of junior doctors during the general surgical induction is required to ensure documentation rates remain high. Further changes to the electronic discharge summary including the use of prewritten text in drop down menus would facilitate more sustainable documentation of advice to patients on discharge.

\section{References}

1 General Medical Council: Good Medical Practice 2013. Available from: http://www.gmc-uk.org/guidance/good_medical_practice (Last accessed 30/01/15).

2 Driver and Vehicle Licensing Agency: DVLA's current medical guidelines for professionals - conditions $D$ to $F$. Available from: https://www.gov.uk/current-medical-guidelines-dvla-guidance-forprofessionals-conditions-d-to-f (Last accessed 10/02/15).

3 Sedgley J, Rickard K, Morris J. A survey of women and health providers about information regarding the timing of driving a car after experiencing a caesarean section. Aust N Z J Obstet Gynaecol 2012 Aug; 52(4): 361-5.

4 Kalkur S, McKenna D, Dobbs SP. 'Doctor - when can I drive?' Advice obstetricians and gynaecologists give on driving after obstetric or gynaecological surgery. Ulster Med J 2007 Sept; 76(3): 141-143.

5 Lewis C, Mauffrey C, Hull P, Brooks S. Knee arthroscopy and driving. Results of a prospective questionnaire survey and review of the literature. Acta Orthop Belg 2011 Jun; 77(3): 336-8.

6 Abbas G, Waheed A. Resumption of car driving after total hip replacement. J Orthop Surg (Hong Kong) 2011 Apr; 19(1): 54-6.

7 Naidu M, Sultan AH, Thakar R. Convalescence advice following gynaecological surgery. J Obstet Gynaecol 2012 Aug; 32(6): 556-9.

8 Royal College of Surgeons: Patients - Recovering from surgery Driving. Available from: http://www.rcseng.ac.uk/patients/recoveringfrom-surgery (Last accessed 10/02/15).

9 Giddins GE, Hammerton A. "Doctor - when can I drive?”: a 


\section{BMJ Quality Improvement Reports}

medical and legal view of the implications of advice on driving after injury or operation. Injury 1999 Sep; 27(7): 495-7.

10 General Medical Council: Good Medical Practice 2013. Domain 1: Knowledge, skills and performance - Record your work clearly, accurately and legibly. Available from: http://www.gmc-

uk.org/guidance/good medical practice/record work.asp (Last accessed 10/02/15)

11 Makela P, Haynes C, Holt K, Kar A. Written medical discharge communication from an acute stroke service: a project to improve content through development of a structured stroke-specific template. BMJ Qual Improv Report 2013; 2: 1.

\section{Declaration of interests}

Nothing to declare.

\section{Ethical approval}

According to the policy activities that constitute research at North Bristol NHS Trust, this work met criteria for operational improvement activities exempt from ethics review. We used the following criteria for determining if improvement activities require ethics review. Policy criterion: The work is primarily intended to improve local care, not provide generalisable knowledge in a field of inquiry. Explanation: The work reported here meets this criterion because provision of advice to patients on discharge is a universally recommended practice. We sought only to evaluate the improvements in compliance with provision of advice as a result of auditing and feedback of compliance rates to hospital staff. 